Received: 2019.12.21 Accepted: 2020.02 .17 Available online: 2020.04 .01

Published: 2020.04.30

\section{Multiple Neural Tube Defects: A Case Report}

Authors' Contribution: Study Design A Data Collection B Statistical Analysis C Data Interpretation D Manuscript Preparation E

Literature Search F Funds Collection G

\author{
AE 1 Mohammad Ahmad Jamous \\ BE 1 Suleiman Shaheer Daoud \\ DF 2 Abdullah Mohammad Abu-Aqoulah
}

1 Department of Neurosurgery - Faculty of Medicine, Jordan University of Science and Technology, Irbid, Jordan

2 Faculty of Medicine, Jordan University of Science and Technology, Irbid, Jordan
Corresponding Author: Conflict of interest:

Patient: $\quad$ Female, newborn

Final Diagnosis: Mutiple neural tube defects

Symptoms: Head and neck mass • neck mass

Medication: -

Clinical Procedure: $\quad$ Staged repair of neural tube defects $\bullet$ V-P shunt for congenital hedrocephalus

Specialty: $\quad$ Neurosurgery

Objective: Congenital defects/diseases

Background: Incomplete closure of the neural tube results in congenital anomalies called neural tube defects (NTD). These defects are rarely multiple, and are characterized by loss of central nervous system soft tissue and bony coverings, along with herniation of the involved part of the CNS through the defect.

Case Report: A newborn female infant was delivered through planned cesarean section due to large occipital encephalocele diagnosed antenatally. The pregnancy was unplanned and the mother did not take folic acid prior to conception. Birth weight was $3.41 \mathrm{Kg}$. Upon delivery, the newborn was healthy, with an Apgar score of 8 . The physical examination revealed 2 large pouches; one was over the occiput, and the other swelling was located over the nape of the neck. Brain MRI revealed large occipital encephalocele and cervical myelomeningocele. The 2 defects were repaired separately, with an uneventful postoperative course.

Conclusions: We report the rare occurrence of multiple NTD. Early repair, either as single or multiple procedures, is mandatory to avoid dramatic complications.

MeSH Keywords: $\quad$ Encephalocele • Meningomyelocele • Neural Tube Defects

Full-text PDF: https://www.amjcaserep.com/abstract/index/idArt/922312

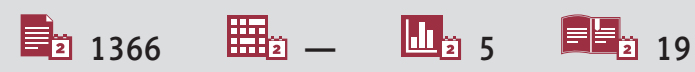




\section{Background}

Neural tube defects (NTD) are defined as heterogeneous and complex congenital abnormalities of the central nervous system, including anencephaly, encephaloceles, and spina bifida. The causes of NTD remain unclear, despite extensive medical research. Contributing factors to NTD occurrence are multifactorial, including interacting complex environmental and genetic elements [1]. Supporting phenomena for the genetic role in NTD etiology include the NTD familial clustering among first- and second-degree relatives of the affected patient, and the association of NTD with many genetics and chromosomal diseases. Nonetheless, the genetics of NTD are clearly complex $[2,3]$. Genetic studies of multiple NTDs are limited by the small number of affected families and members, the high perinatal death rate, and the NTD patient's low reproduction.

Various environmental risk factors are highly associated with NTD, including, but not limited to, socioeconomic status, occupations such as foods industries, nursing, farming, and use of pesticides, and potential workplace exposures to hazards such as ionizing radiation, viruses, organic solvents, anesthetic agents, and paints [4-6]. Maternal co-morbid conditions such as diabetes and obesity [7] and hyperthermia during pregnancy are postulated to increase the risk of a fetuses having NTD (odds ratio 1.95) [8]. The maternal nutritional status is known to alter fetal growth and development, and folic acid supplements during pregnancy are known to reduce the risk of NTD by $50 \%$ to $70 \%$ [9].

The coexistence of myelomeningocele and encephalocele is extremely rare and it occurs in less than $1 \%$ of NTD cases. [10]. The combination of these defects has been explained by the multisite neural tube closure theory, which hypothesizes that neural tube closure occurs at multiple sites during the embryogenesis, and failure of closure of these sites will produce NTD [11].

A recent literature review found 48 reported cases of multiple NTDs in the same patient in the past 50 years [12]. Among those cases, there were only 5 cases reported as double encephalocele [12-16], and 3 reported cases of both occipital encephalocele with suboccipital or cervicothoracic myelomeningocele $[11,17]$. Here, we report a case of both occipital encephalocele with cervical myelomeningocele, and discuss the multisite theory for neural tube closure.

\section{Case Report}

A newborn female infant of a 42-year-old mother and a 61-yearold father was delivered through planned cesarean section due to large occipital encephalocele diagnosed antenatally after full-term pregnancy. The newborn was the last sibling of 5 ; her 4 brothers were delivered normally without any history of congenital defects. The pregnancy was unplanned and the mother did not take folic acid prior to conception, but she was on regular folic acid supplement during the first 3 months of pregnancy. The mother denied exposure to radiation or heat during pregnancy; she also denied taking anticonvulsant medication. She is not diabetic or obese. Birth weight was $3.41 \mathrm{Kg}$.

Upon delivery, the newborn was healthy, with an Apgar score of 8 . The physical examination revealed 2 large, soft, dark bluecolored pouches; one was over the occiput, and the other swelling was located over the nape of the neck (Figure 1). There was no clinical evidence of other NTDs along the spinal column.

Magnetic resonance imagining (MRI) demonstrated a large posterior occipital encephalocele measuring $4.1 \times 3.4 \mathrm{~cm}$, with herniation of posterior parts of the occipital lobes and cerebellum through the encephalocele defect (Figure 2). This occipital encephalocele was accompanied with suboccipital cervical myelomeningocele measuring $5 \times 2.9 \mathrm{~cm}$, and contained the herniated inferior cerebellar vermis, cerebellar tonsils, and upper cervical cord (Figure 2). MRI of the spinal cord revealed syringomyelia involving the cervicothoracic cord (Figure 3). The overall findings represent double NTD with Chiari malformation type III. Cerebral CT venography was done prior to surgery to exclude the presence of major venous component within the defect (Figure 4).

The 2 defects were repaired separately; the encephalocele defect was repaired in the first surgery. During Encephalocele surgery repair, the herniated gliotic cerebellar tissue in the occipital encephalocele was amputated, and the dural defect was closed in a watertight fashion, while the skull defect was closed using flexible titanium mesh. Upper cervical myelomeningocele repair was done 1 week after repair of the occipital encephalocele. During myelomeningocele repair, a minimally vascular dysplastic tissue with intact cerebellar lobes and tonsils was seen in the herniated sac. The dysplastic mass was totally excised and a dura flap was created and closed tightly. The surgical repair of the NTD was uneventful.

One week later, the patient underwent right frontal ventriculo-peritoneal shunt insertion surgery for congenital hydrocephalus. The baby was followed up for 8 months and showed mild developmental delay in motor and cognitive milestones. The surgical site is dry and clean, and head circumference was $40 \mathrm{~cm}$. The baby did not have feeding or breathing difficulties. Figure 5 shows the postoperative brain CT scan. 


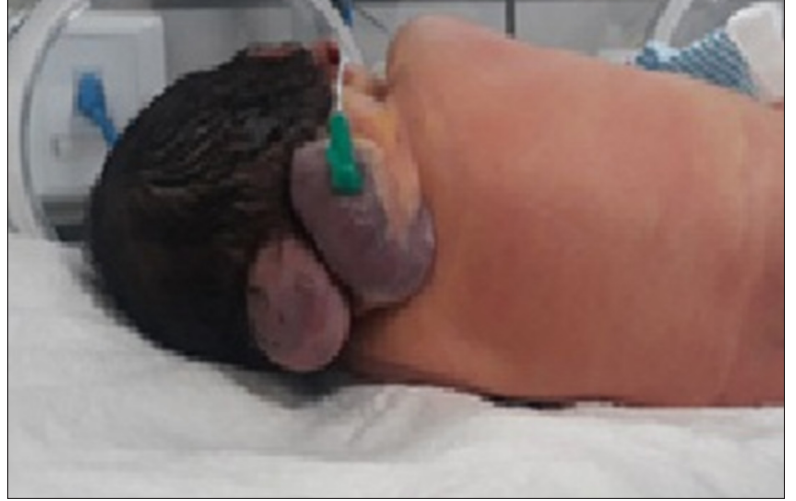

Figure 1. Photograph of the newborn showing 2 large, soft, dark blue-colored pouches; one was over the occiput, and the other swelling was located over the nape of the neck.

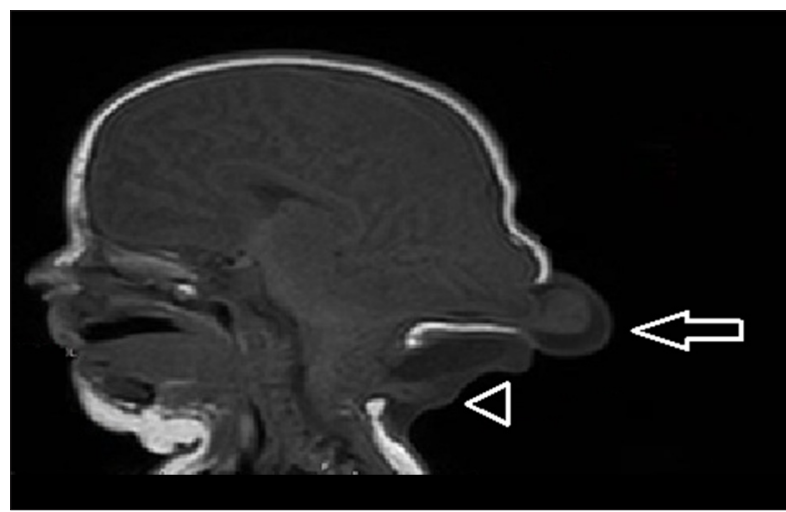

Figure 2. Brain MRI, T1-weighted image, sagittal view showing large posterior occipital encephalocele measuring $4.1 \times 3.4 \mathrm{~cm}$ (arrow) with herniation of posterior parts of occipital lobes and cerebellum through the encephalocele defect associated with cervical myelomeningocele measuring $5 \times 2.9 \mathrm{~cm}$ (arrow head) containing the herniated inferior cerebellar vermis, cerebellar tonsils, and upper cervical cord.

\section{Discussion}

The existence of multiple neural tube defects in one patient has been rarely reported in the literature. The exact worldwide incidence is still unknown [10]. In this case, we report 2 neural tube defects found in a single patient - encephalocele and cervical myelomeningocele. The main risk factor for NTD occurrence in this baby was the late supplementation of folic acid during pregnancy. The mother denied exposure to environmental hazards that can increase the risk for NTD; similarly, there was no family history of NTD in the first- or second-degree relatives. Because of the high morbidity and mortality rates of NTD infants, even with surgical and medical care, prevention is essential. In Jordan, preconception folic acid supplementation is the main preventive measure for

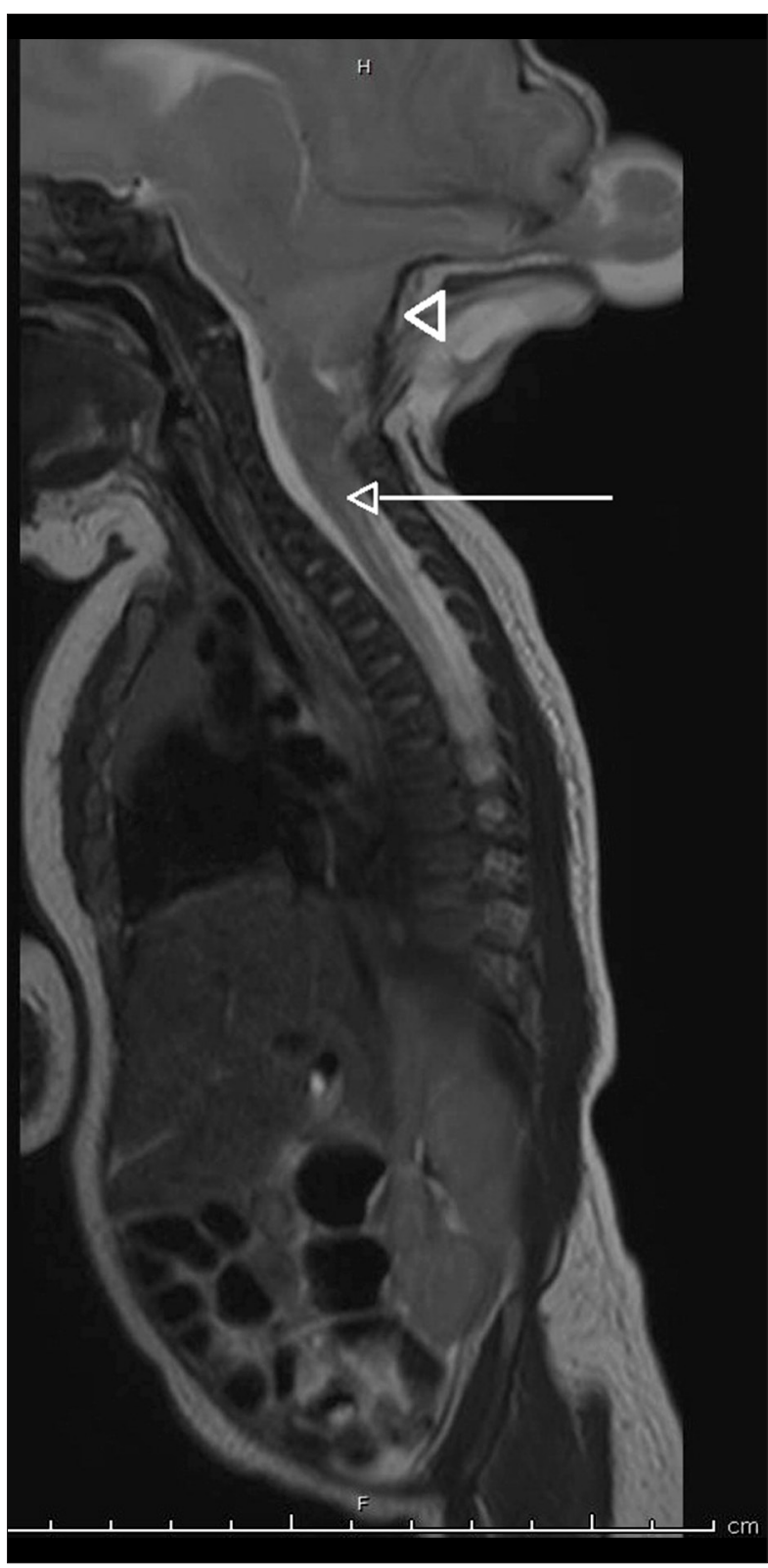

Figure 3. Whole-spine MRI, T2-weighted image, sagittal view showing Chiari III malformation (arrow head) and cervicothoracic syringomyelia (arrow).

NTD. Early prenatal diagnosis and termination is currently legally accepted. Unfortunately, fortification of staple food is not mandatory in Jordan.

The most popular theory explaining neural tube defects formation is the multisite neural tube closure theory [11], which hypothesizes that there are 5 sites of closure of the neural tube during embryogenesis, and any failure of closure at any site will produce an NTD at that site [12]. 


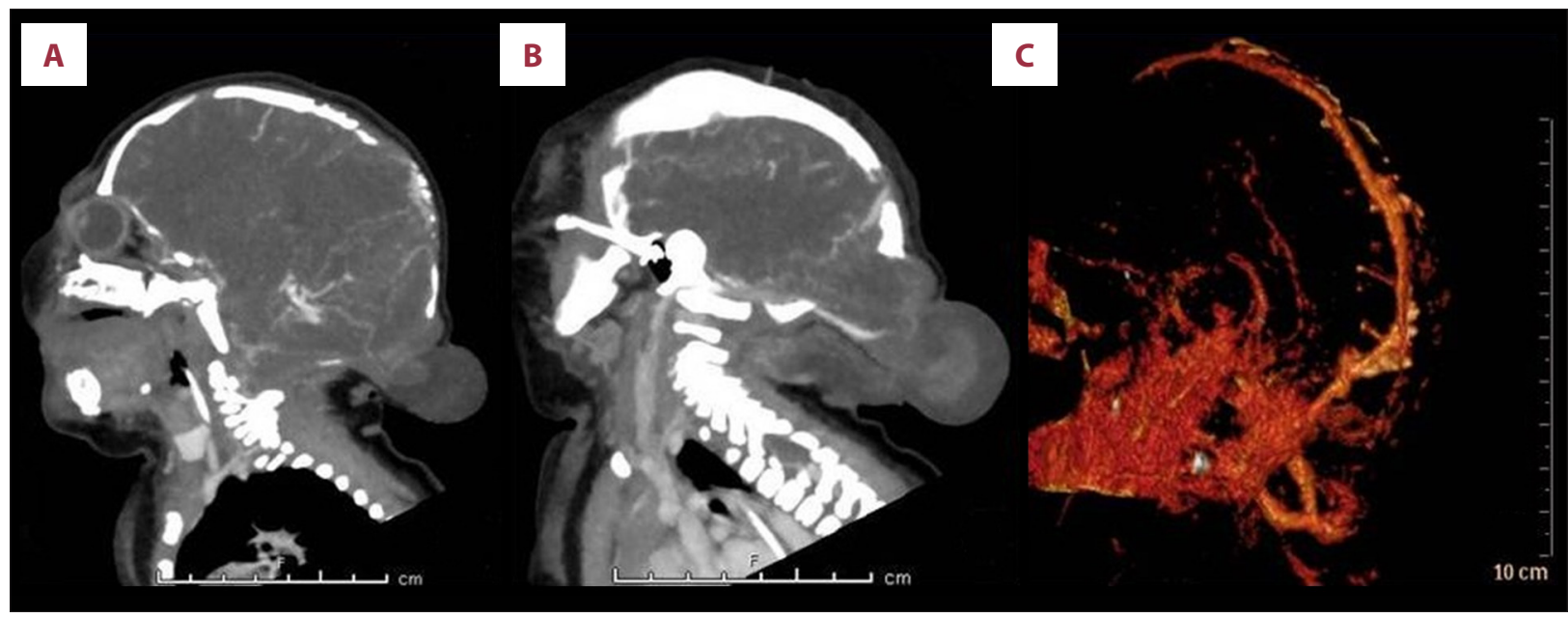

Figure 4. (A-C) Cerebral CT venography demonstrating the absence of a major venous component within the defect.

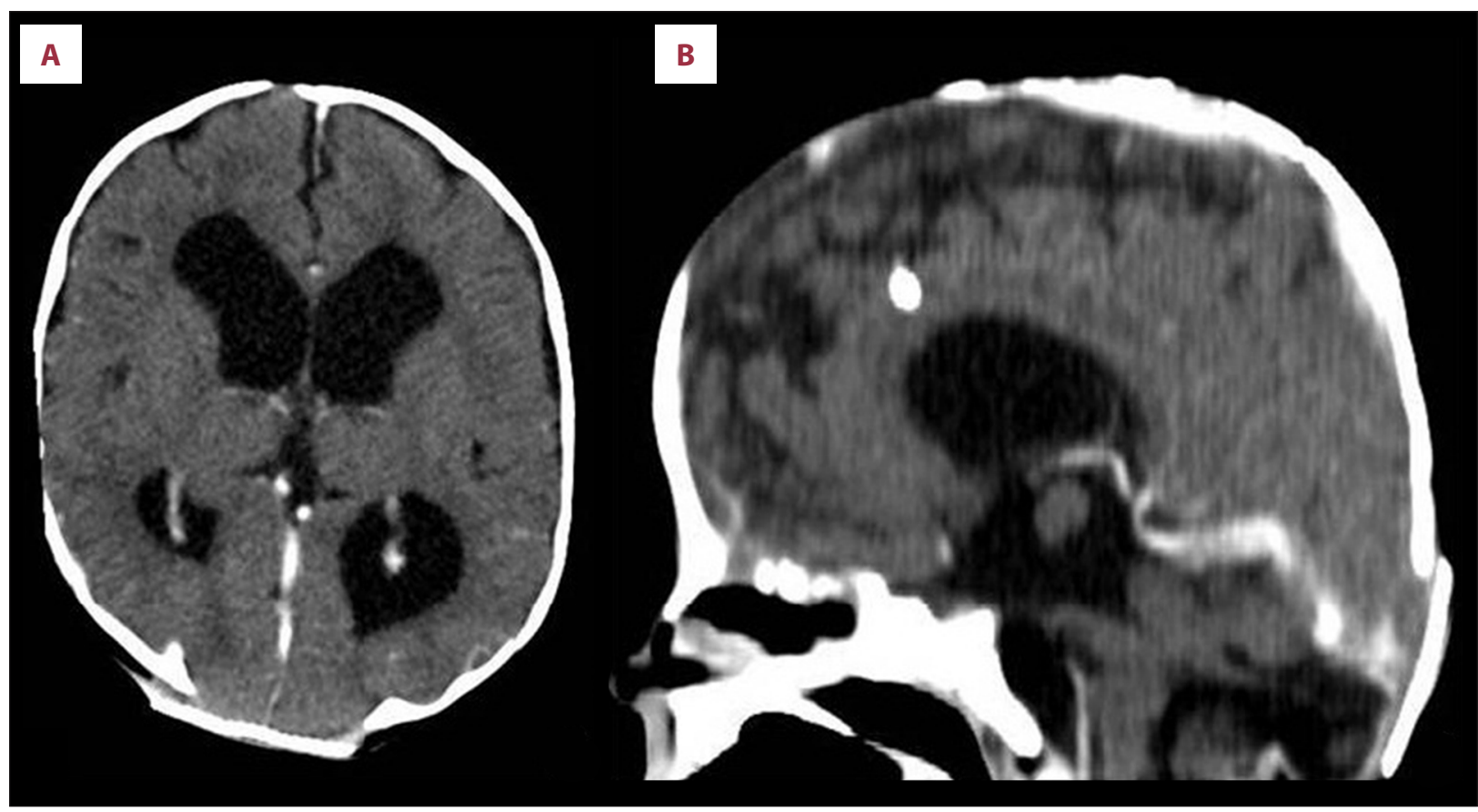

Figure 5. (A, B) Showing axial and sagittal postoperative brain CT scan, respectively.

Another theory of formation of NTDs is the "single-site" theory, which hypothesizes that the closure starts as a "zipper" at the midcervical region and progresses bidirectional to the most caudal and rostral site.

Although the number of published cases on multiple NTDs is limited, the occurrence of some cases of multiple NTD along the longitudinal axis supports the multisite hypothesis. The single-site theory fails to explain these multiple NTDs, as it postulates that the NTD should be at the most rostral and caudal ends, which was not seen in these cases $[12,13]$.
The timing of NTD surgical repair is controversial [14]. However, early closure of the myelomeningocele provides a better prognosis for neurogenic bladder in children, and prevents CSF infection and further neurological deterioration $[15,16]$. The prognosis of surgically treated occipital encephalocele depends on the presence of brain tissue in the lesion. Usually the herniated parts of the brain through the encephalocele defect are non-viable cerebral tissue, and excision of this herniated part would not result in new neurological deficits.

There is no consensus on whether to repair multiple NTDs in one surgery or to do it in multiple surgeries; similarly, there is no consensus on which NTD to operate on first in cases of 
multiple NTDs. We preferred multiple surgeries for this newborn to reduce the risks of prolonged surgery and blood loss in this fragile patient. In cases of multiple NTDs, we propose to start closure of the ruptured, larger, or most cranial NTD. Closure of multiple NTD in the same surgery increases the risk of surgical morbidities. Hydrocephalus is usually present in newborn with multiple NTDs. The baby in the present report underwent VP shunting following repair of the NTDs, as his hydrocephalic symptoms and signs developed following NTD repair.

In this report, we present a case of both cervical myelomeningocele and occipital encephalocele in the same patient. The radiological finding showed Chiari malformation type III. Though the surgical repair of these NTDs was uneventful, we believe that the overall prognosis of patients with Chiari malformation type III is poor due to respiratory failure $[18,19]$.

\section{References:}

1. Padmanabhan R: Etiology, pathogenesis and prevention of neural tube defects. Congenit Anom (Kyoto), 2006; 46(2): 55-67

2. Volcik KA, Zhu H, Shaw GM et al: Apolipoprotein E and apolipoprotein B genotypes and risk for spina bifida. Teratology, 2002; 66: 257-59

3. Juriloff DM, Harris MJ: Mouse models for neural tube closure defects. Hum Mol Genet, 2000; 9: 993-1000

4. Brender JD, Suarez L: Paternal occupation and anencephaly. Am J Epidemiol, 1990; 131: 517-21

5. Matte TD, Mulinare J, Erickson JD: Case-control study of congenital defects and parental employment in health care. Am J Ind Med, 1993; 24: 11-23

6. Sever LE: Looking for causes of neural tube defects: Where does the environment fit in? Environ Health Perspect, 1995; 103(Suppl. 6): 165-71

7. Ray JG, Vermeulen MJ, Meier C, Wyatt PR: Risk of congenital anomalies detected during antenatal serum screening in women with pregestational diabetes. QJM, 2004; 97: 651-53

8. Moretti ME, Bar-Oz B, Fried S, Koren G: Maternal hyperthermia and the risk for neural tube defects in offspring: Systematic review and meta-analysis. Epidemiology, 2005; 16: 216-19

9. MRC Vitamin Study Research Group Prevention of neural tube defects: Results of the medical research council vitamin study. Lancet, 1991; 338: 131-37

10. Tungaria A, Srivastav AK, Mahapatra AK, Kumar R: Multiple neural tube defects in the same patient with no neurological deficit. J Pediatr Neurosci, 2010; 5(1): 52-54

\section{Conclusions}

The current report of multiple NTD in the same newborn supports the multisite closure theory of the neural tube. Early repair, either as single or multiple procedures, is mandatory to avoid the complications related to these defects.

\section{Conflict of interest}

None.

11. Ahmad FU, Dwarakanath S, Sharma BS, Mahapatra AK: Multiple neural tube defects: A clinical series of seven cases and their embryological basis. Pediatr Neurosurg, 2008; 44(4): 280-87

12. Deora H, Srinivas D, Shukla D et al: Multiple-site neural tube defects: Embryogenesis with complete review of existing literature. Neurosurg Focus, 2019; 47(4): E18

13. Goyal PK, Singh D, Singh H, Tandon M: Suboccipital double barrel twin meningocoele: Another new theory? J Pediatr Neurosci, 2010; 5(2): 126-28

14. Ramdurg SR, Gubbi S, Odugoudar A, Kadeli V: A rare case of split pons with double encephalocoele, dermal sinus tract, and lipomeningomyelocele: A case report and review of literature. Childs Nerv Syst, 2014; 30(1): 173-76

15. Canaz H, Ayçiçek E, Akçetin MA et al: Supra- and infra-torcular double occipital encephalocele. Neurocirugia (Astur), 2015; 26(1): 43-47

16. Singh DK, Singh N, Kumar P: Double suboccipital meningoencephalocele: A unique case report. Pediatr Neurosurg, 2012; 48(5): 331-32

17. Srinivas D, Sharma BS, Mahapatra AK: Triple neural tube defect and the multisite closure theory for neural tube defects: Is there an additional site? Case report. J Neurosurg Pediatr, 2008; 1(2): 160-63

18. Van Allen MI, Kalousek DK, Chernoff GF et al: Evidence for multi-site closure of the neural tube in humans. Am J Med Genet, 1993; 47(5): 723-43

19. Ahmad FU, Dwarakanath S, Sharma BS, Mahapatra AK: Multiple neural tube defects: A clinical series of seven cases and their embryological basis. Pediatr Neurosurg, 2008; 44(4): 280-87 